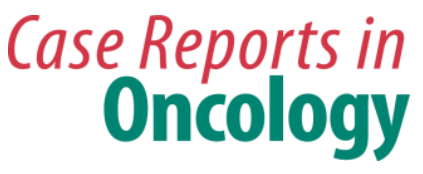

Case Rep Oncol 2016;9:586-592

DOI: $10.1159 / 000449448$

Published oniıne: Uctober 12, 2016

(C) 2016 The Author(s)

Published by S. Karger AG, Basel

www.karger.com/cro

This article is licensed under the Creative Commons Attribution-NonCommercial 4.0 International License (CC BY-NC) (http://www.karger.com/Services/OpenAccessLicense).

Usage and distribution for commercial purposes requires written permission.

\title{
Intrathecal Methotrexate and Craniospinal Radiotherapy Can Be an Effective Treatment of Carcinomatous Meningitis in Patients with Breast Cancer: Case Reports
}

\author{
Magdalena Meissner ${ }^{a} \quad$ Alfredo Addeo ${ }^{b}$ \\ ${ }^{a}$ Institute of Medical Genetics, Cardiff University, Cardiff, UK; ${ }^{b}$ Bristol Cancer Institute, \\ University Hospitals Bristol Foundation NHS Trust, Bristol, UK
}

\section{Keywords}

Breast cancer - Leptomeningeal carcinoma - Meningeal carcinomatosis · Carcinomatous meningitis · Intrathecal methotrexate $\cdot$ Craniospinal radiotherapy

\begin{abstract}
Introduction: Carcinomatous meningitis in breast cancer occurs as a complication in up to $5 \%$ of all cases. It is a very devastating diagnosis, with a median patient survival of about 3 months. Treatment is very controversial, and different modalities of treatment have been used but none of them show significant benefit for overall survival. Case Reports: We report 2 cases of carcinomatous meningitis in breast cancer patients. They received a similar treatment of a combination of intrathecal (IT) methotrexate followed by craniospinal radiotherapy. Both patients survived for many years after treatment and are in complete clinical and radiological remission. Conclusion: Meningeal metastasis from breast cancer can be very effectively treated with IT and/or systemic chemotherapy followed by craniospinal radiotherapy. Further studies are needed to determine the effectiveness of this sequential combination of chemotherapy with radiotherapy.

2016 The Author(s)




\section{Introduction}

Carcinomatous meningitis, also called 'neoplastic meningitis', 'leptomeningeal carcinoma', or 'meningeal carcinomatosis', is a very rare manifestation of metastatic cancer caused by the infiltration of cancer cells into the central nervous system through cerebrospinal fluid [1]. The diagnosis is confirmed by imaging of the nervous system and cerebrospinal fluid analysis. Carcinomatous meningitis in breast cancer occurs as a complication in up to $5 \%$ of all cases [2]. It is a very devastating diagnosis, with a median patient survival of about 3 months [2]. The treatment is very controversial. From a literature review, different modalities of treatment have been used, such as intrathecal (IT) and/or systemic chemotherapy and radiotherapy, but none of them show significant benefit for overall survival [2].

We present 2 patients who have survived for many years after treatment with IT methotrexate and subsequent craniospinal radiotherapy. To our knowledge, these are the first patients who have survived for that long.

\section{Case 1}

In October 2000, a 62-year-old with a background of breast cancer was transferred from the Neurosciences Unit to the Bristol Oncology Unit with right 7th nerve palsy and numbness with pain affecting the left-sided chest and abdomen. Clinical examination confirmed lower motor neuron 7th nerve palsy and altered sensation of the left chest wall and abdomen but no clinical evidence of breast cancer recurrence.

Magnetic resonance imaging (MRI) of the brain and spine did not provide an explanation for her symptoms, but pathological examination of the cerebrospinal fluid revealed numerous malignant cells consistent with carcinomatous meningitis.

In 1992, the patient was diagnosed with T2 grade 2 intraductal carcinoma, with no lymph nodes identified at histology spaceman. No receptors were available at the time of diagnosis. Unfortunately, in those years, hormonal receptor status was not a standard practice, and the patient was put on tamoxifen for 2 years.

Staging computed tomography (CT) of the chest/abdomen/pelvis showed liver haemangiomata, which was also confirmed on ultrasound, without evidence of metastatic disease. A bone scan showed a solitary hot spot at the L1 level, which turned out to be degenerative change.

On transfer to the Oncology Unit she was comfortable as her pain was controlled with morphine and ibuprofen. However, she was very anxious and pessimistic. As a retired midwife, she had some understanding of the implication of her diagnosis. Her performance status was 2 .

The most likely diagnosis was localized meningeal recurrence of breast cancer. She was commenced on dexamethasone and tamoxifen. Steroids improved her condition so she could receive IT methotrexate at a dose of $12.5 \mathrm{mg}$ once weekly over 6 weeks. After a break of 1 month, she commenced craniospinal radiotherapy - 36 Gy in 20 fractions over 4 weeks. Her main symptoms had improved after IT chemotherapy. She tolerated her treatment quite well, with no major side effects.

She was last seen in March 2016 and is due to be seen again in March next year, so she has been alive for 17 years since she had been diagnosed with carcinomatous meningitis. At the age of 70 years, she was able to walk a mile and dance. Currently, she is 77 and has been having some short memory problems, and her mobility is gradually deteriorating. 


\section{Case 2}

In September 2008, a 43-year-old woman presented to the Emergency Department at Frenchay Hospital with a 3-month history of back pain, right hip and knee pain, right leg weakness, constipation and urine incontinence, altered sensation in the limbs, and visual disturbance with flashing lights in the left eye. Two weeks prior to this admission she was seen at Frenchay Hospital with cauda equina symptoms. She has had an MRI of the spine, which showed a disc prolapse but did not explain the symptoms. The patient was discharged. However, her pain continued to worsen, and sensation changes progressed across the whole of the perianal region. She was unable to sit down for 8 weeks due to pain on flexion and started using a stick as her right leg was weak.

On examination she had a decreased sensation in the perianal area. Neurological examination of the upper limbs revealed a slight numbness of the radial side of the forearm. Neurological examination of the lower limbs revealed the following: reduced power in the right hip flexion (1/5) and extension $(2 / 5)$, the power in the left leg was $4 / 5$, absent reflexes in both legs, reduced sensation in the right medial aspect of the distal right calf dorsum of the foot sole and the lateral dorsum of the left foot and the sole of the left foot. On examination of her visual fields, she demonstrated a loss of outer vision in her left eye.

CT and MRI of the head showed metastases in the right parietal occipital region, together with some smaller metastases associated with the dura and skull base (fig. 1). MRI of the spine revealed fairly diffuse infiltrating nodules in the nerve roots of the lumbosacral plexus. Her performance status was 3 due to progressive leg weakness.

The diagnosis was meningeal carcinomatosis with brain metastasis from breast cancer. No other signs of metastatic breast cancer were seen on CT at this point.

A year prior to presentation, she had been diagnosed with $4 \times 3 \mathrm{~cm}$ grade 3 triplenegative invasive ductal carcinoma treated with 6 cycles of neoadjuvant TAC chemotherapy followed by wide local excision and axillary node clearance. None of the 10 lymph nodes showed viable cancer. She had also completed radiotherapy to her left breast in May 2008.

The patient was transferred to our centre and started on inpatient IT methotrexate 12.5 mg with oral capecitabine. The first 4 cycles of IT methotrexate were administered twice weekly due to deteriorating symptoms, as her left leg become weak as well. Her vision improved after 1 cycle of treatment. Other neurological symptoms improved after 3 cycles. From that point on she received weekly IT methotrexate. The 6th cycle was delayed by a week due to urosepsis and opioid toxicity. Cerebrospinal fluid was sent for analysis by cytospin before each IT treatment. She was discharged on capecitabine (1 week on and 1 week off at a $75 \%$ dose) before cycle 7 of IT methotrexate. A further cycle of IT methotrexate was not given as she was readmitted with pyrexia and pain in her lower abdomen, perianal area, and shouting/burning pains down the leg. She did not require any antibiotics, and the pain was controlled with analgesia. Her symptoms improved and she received cycle 8 of IT methotrexate as inpatient. In total, she received 8 cycles of IT methotrexate, and her neurological symptoms have improved.

Malignant cells were still present in the cerebrospinal fluid. MRI of the head and spine after IT chemotherapy combined with capecitabine showed good response to therapy with disappearance of some of the smaller metastatic lesions and a significant reduction in the size of the larger lesions in the brain, but the spine lesions within the theca were more prominent, suggesting progressive disease.

She was referred for craniospinal radiotherapy and started 10 days after her last IT treatment. She received 36 Gy in 20 fractions over 4 weeks. MRI of the head/spine after ra- 


\section{Case Reports in Oncology}

diotherapy showed significant improvement in the appearances of both intracerebral and ependymal metastases.

In April 2009, MRI of the head showed further involution of cerebral metastases. The posterior fossa and right frontal metastases were essentially invisible, while the right occipital metastases in the lobe showed further involution. There was no convincing focal meningeal mass. She was treated with carboplatin 6 months after completion of craniospinal radiotherapy to prevent recurrence of meningeal disease. She completed 4 cycles of carboplatin between 21/05/2009 and 10/09/2009.

In 2011, she had recurrent solitary right occipital metastasis, which was treated with stereotactic radiotherapy. In 2012, MRI showed increasing occipital abnormality with oedema, which was surgically excised and proved to be radiation necrosis only, with no viable tumour. In 2013, MRI showed no new disease, only post-surgical changes (fig. 2).

It has been 8 years since she first presented with neurological symptoms. Currently, the patient is in complete clinical and radiological remission and has recently been discharged from our clinic.

\section{Discussion}

We present 2 patients with a history of breast cancer who developed meningeal carcinomatosis with no evidence of metastatic visceral disease. Both patients were treated with IT methotrexate and subsequent craniospinal radiotherapy. The second patient has had further treatment for brain metastasis and also received concomitant oral capecitabine with IT methotrexate. The first patient is still alive after 17 years and the second patient 8 years after primary treatment. To our knowledge, there are no patients in the literature who have survived that long after meningeal carcinomatosis diagnosis.

Still, treatment remains controversial. There are National Comprehensive Cancer Network (NCCN) guidelines how to treat meningeal disease according to 'poor-risk' and 'goodrisk' groups. Many patients will fall in between these two groups. Clinical judgment normally dictates how intensive the treatment should be. The recommendation for the 'poor-risk' group is supportive care. External beam radiotherapy can be considered for symptomatic sites. The options for 'good-risk' patients and those with breast cancer are IT chemotherapy, high-dose methotrexate, or craniospinal radiotherapy [3].

In the literature, there is various evidence of different types of treatments, such as systemic chemotherapy of capecitabine, eribulin as a single treatment, or combined with IT chemotherapy using methotrexate, topotecan, thiothepa, and liposomal cytarabine. There are also some reports of using craniospinal radiotherapy. Overall survival in those studies was counted in months $[2,4-8]$.

We can conclude that isolated meningeal metastasis from breast cancer can potentially be very effectively treated with combined IT and/or systemic chemotherapy followed by craniospinal radiotherapy. Further studies are needed to determine the effectiveness of this combined treatment of chemotherapy with radiotherapy.

\section{Statement of Ethics}

The authors have no ethical conflicts to disclose. 


\section{Disclosure Statement}

The authors repot no conflicts of interest.

\section{References}

1 Duan H, Li M, Sun X: Clinical features of patients with carcinomatous meningitis in the Chinese population: report of 4 cases and review of the literature. Turk Neurosurg 2014;24:13-18.

-2 de Azevedo CR, Cruz MR, Chinen LT, Peres SV, Peterlevitz MA, de Azevedo Pereira AE, Fanelli MF, Gimenes DL: Meningeal carcinomatosis in breast cancer: prognostic factors and outcome. J Neurooncol 2011;104:565-572.

3 Network NCCN: NCCN Clinical Practice Guidelines in Oncology (NCCN Guidelines ${ }^{\circledR}$ ). Central Nervous System Cancers, in NCCN Clinical Practice Guidelines in Oncology, 2015.

4 Jayson GC, Howell A, Harris M, Morgenstern G, Chang J, Ryder WD: Carcinomatous meningitis in patients with breast cancer. An aggressive disease variant. Cancer 1994;74:3135-3141.

-5 Le Rhun E, Taillibert S, Zairi F, Kotecki N, Devos P, Mailliez A, Servent V, Vanlemmens L, Vennin P, Boulanger T, Baranzelli MC, Andre C, Marliot G, Cazin JL, Dubois F, Assaker R, Bonneterre J, Chamberlain MC: A retrospective case series of 103 consecutive patients with leptomeningeal metastasis and breast cancer. J Neurooncol 2013;113:83-92.

-6 Salgia S, Fleming GF, Lukas RV: Leptomeningeal carcinomatosis from breast cancer treated with intrathecal topotecan with concomitant intravenous eribulin. J Clin Neurosci 2014;21:1250-1251.

7 Comte A, Jdid W, Guilhaume MN, Kriegel I, Piperno-Neumann S, Dieras V, Dorval T, Pierga JY, Cottu PH, Mignot L, Bidard FC: Survival of breast cancer patients with meningeal carcinomatosis treated by intrathecal thiotepa. J Neurooncol 2013;115:445-452.

8 Gauthier H, Guilhaume MN, Bidard FC, Pierga JY, Girre V, Cottu PH, Laurence V, Livartowski A, Mignot L, Dieras V: Survival of breast cancer patients with meningeal carcinomatosis. Ann Oncol 2010;21:21832187. 


\section{Case Reports in Oncology}

\begin{tabular}{l|l}
\hline Case Rep Oncol 2016;9:586-592 \\
\hline DOI: 10.1159/000449448 & $\begin{array}{l}\text { @ 2016 The Author(s). Published by S. Karger AG, Basel } \\
\text { www.karger.com/cro }\end{array}$ \\
\hline
\end{tabular}

Meissner and Addeo: Intrathecal Methotrexate and Craniospinal Radiotherapy Can Be an Effective Treatment of Carcinomatous Meningitis in Patients with Breast Cancer

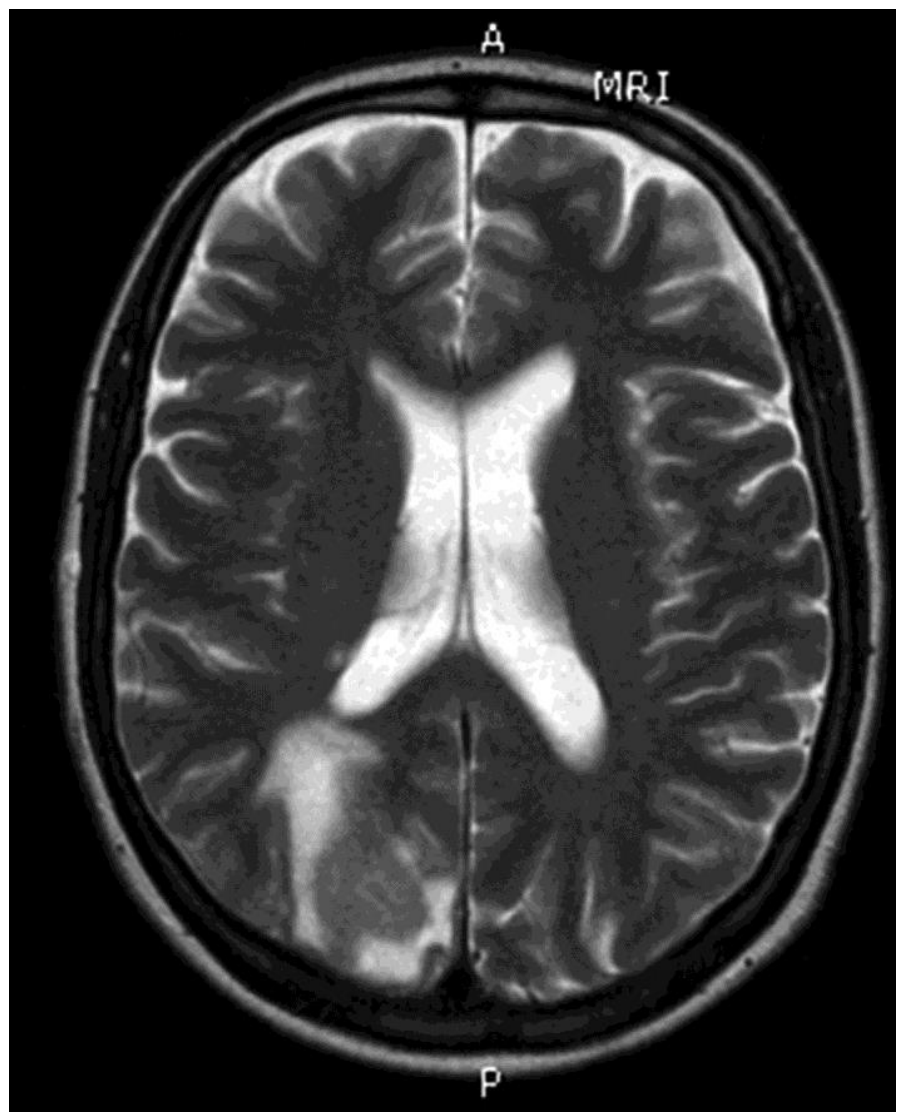

Fig. 1. MRI of the patient's head at diagnosis in the year 2008. Metastatic lesion in the right occipital region is shown. 


\section{Case Reports in Oncology}

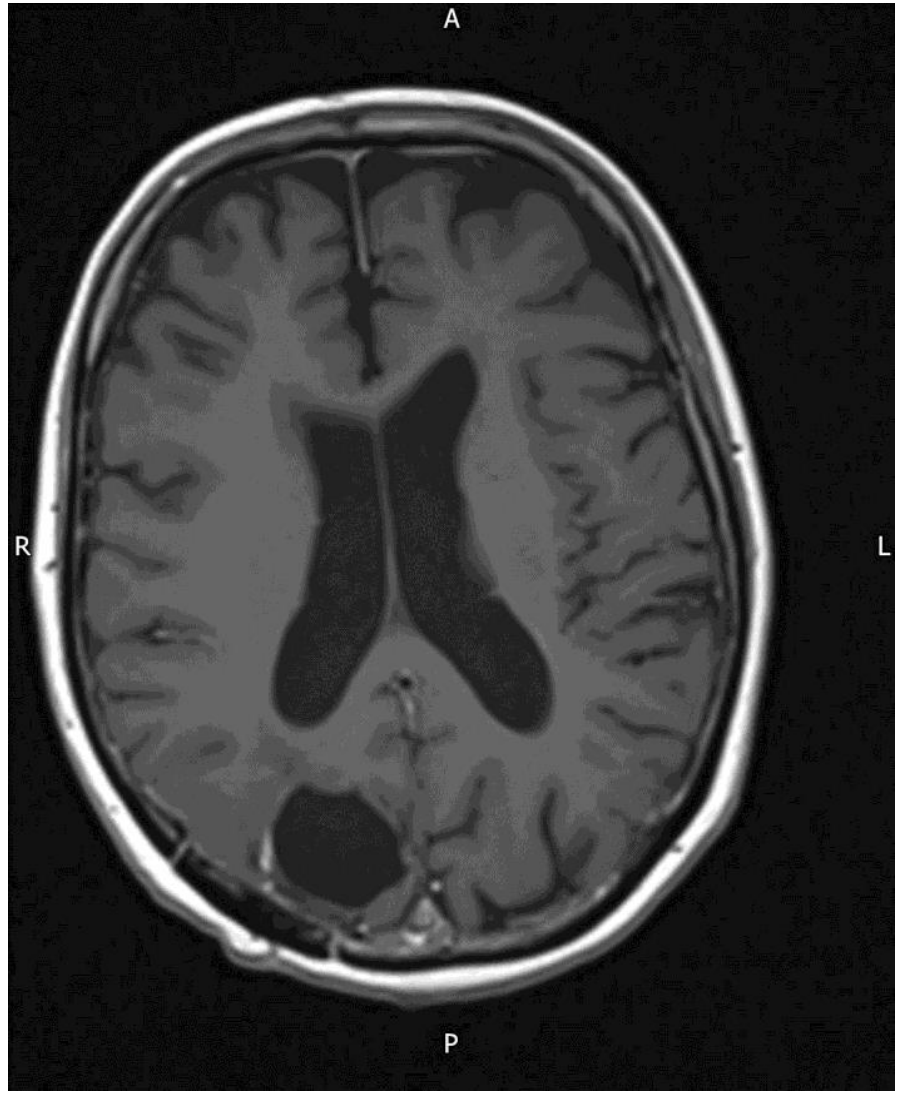

Fig. 2. MRI of the patient's head in 2013. Post-surgical change in the right occipital region is shown.

Meissner and Addeo: Intrathecal Methotrexate and Craniospinal Radiotherapy Can Be an Effective Treatment of Carcinomatous Meningitis in Patients with Breast Cancer

st-surgical change in the right occipital region is shown. 